\title{
Common Sense Reasoning for Detection, Prevention, and Mitigation of Cyberbullying
}

\author{
KARTHIK DINAKAR, BIRAGO JONES, CATHERINE HAVASI, HENRY LIEBERMAN, \\ and ROSALIND PICARD, MIT Media Lab
}

Cyberbullying (harassment on social networks) is widely recognized as a serious social problem, especially for adolescents. It is as much a threat to the viability of online social networks for youth today as spam once was to email in the early days of the Internet. Current work to tackle this problem has involved social and psychological studies on its prevalence as well as its negative effects on adolescents. While true solutions rest on teaching youth to have healthy personal relationships, few have considered innovative design of social network software as a tool for mitigating this problem. Mitigating cyberbullying involves two key components: robust techniques for effective detection and reflective user interfaces that encourage users to reflect upon their behavior and their choices.

Spam filters have been successful by applying statistical approaches like Bayesian networks and hidden Markov models. They can, like Google's GMail, aggregate human spam judgments because spam is sent nearly identically to many people. Bullying is more personalized, varied, and contextual. In this work, we present an approach for bullying detection based on state-of-the-art natural language processing and a common sense knowledge base, which permits recognition over a broad spectrum of topics in everyday life. We analyze a more narrow range of particular subject matter associated with bullying (e.g. appearance, intelligence, racial and ethnic slurs, social acceptance, and rejection), and construct BullySpace, a common sense knowledge base that encodes particular knowledge about bullying situations. We then perform joint reasoning with common sense knowledge about a wide range of everyday life topics. We analyze messages using our novel AnalogySpace common sense reasoning technique. We also take into account social network analysis and other factors. We evaluate the model on real-world instances that have been reported by users on Formspring, a social networking website that is popular with teenagers.

On the intervention side, we explore a set of reflective user-interaction paradigms with the goal of promoting empathy among social network participants. We propose an "air traffic control"-like dashboard, which alerts moderators to large-scale outbreaks that appear to be escalating or spreading and helps them prioritize the current deluge of user complaints. For potential victims, we provide educational material that informs them about how to cope with the situation, and connects them with emotional support from others. A user evaluation shows that in-context, targeted, and dynamic help during cyberbullying situations fosters end-user reflection that promotes better coping strategies.

Categories and Subject Descriptors: H.1.2 [Models and Principles]: User/Machine Systems-Human information processing; 1.5.1 [Pattern Recognition]: Models

General Terms: Algorithms, Design

Additional Key Words and Phrases: Common sense reasoning, affective computing, artificial intelligence

ACM Reference Format:

Dinakar, K., Jones, B., Havasi, C., Lieberman, H., and Picard, R. 2012. Common sense reasoning for detection, prevention, and mitigation of cyberbullying. ACM Trans. Interact. Intell. Syst. 2, 3, Article 18 (September 2012), 30 pages.

DOI $=10.1145 / 2362394.2362400$ http://doi.acm.org/10.1145/2362394.2362400

The reviewing of this article was managed by associate editor Oliviero Stock.

Author's address: K. Dinakar (corresponding author); email: karthik@media.mit.edu.

Permission to make digital or hard copies of part or all of this work for personal or classroom use is granted without fee provided that copies are not made or distributed for profit or commercial advantage and that copies show this notice on the first page or initial screen of a display along with the full citation. Copyrights for components of this work owned by others than ACM must be honored. Abstracting with credit is permitted. To copy otherwise, to republish, to post on servers, to redistribute to lists, or to use any component of this work in other works requires prior specific permission and/or a fee. Permission may be requested from Publications Dept., ACM, Inc., 2 Penn Plaza, Suite 701, New York, NY 10121-0701, USA, fax +1 (212) 869-0481, or permissions@acm.org.

(c) 2012 ACM 2160-6455/2012/09-ART18 $\$ 15.00$

DOI 10.1145/2362394.2362400 http://doi.acm.org/10.1145/2362394.2362400 


\section{THE PROBLEM OF CYBERBULLYING}

Cyberbullying has grown as a major problem in recent years, afflicting children and young adults. Recent surveys on the prevalence of cyberbullying have shown that almost $43 \%$ of teens in the United States alone were subjected to cyberbullying at some point [Ybarra 2010]. The adverse impact that cyberbullying has on victims, especially adolescents in their formative years, is well documented [Vandebosch and Cleemput 2009]. The American Academy of Child and Adolescent Psychiatry says that targets of cyberbullying often deal with significant emotional and psychological suffering [Menesini and Nocentini 2009]. Studies have shown that cyber-victimization and cyberbullying on social networks involving adolescents are strongly associated with psychiatric and psychosomatic disorders [Sourander et al. 2010]. Children whose coping mechanisms may not be as strong as that of an adult sometimes suffer grievously, sometimes resulting in tragic outcomes like self-injurious behavior and even suicides.

According to the National Crime Prevention Council, cyberbullying can be defined as the following: "when the Internet, cell phones or other devices are used to send or post text or images intended to hurt or embarrass another person"1. Social scientists such as Danah Boyd [Boyd 2007] have described four aspects of the Web that change the very dynamics of bullying and magnify it to new levels: persistence, searchability, replicability and invisible audiences.

Cyberbullying is a more persistent version of traditional forms of bullying, extending beyond the physical confines of a school, sportsfield, or workplace, with the victim often experiencing no respite from it. Cyberbullying gives a bully the power to embarrass or hurt a victim before an entire community online, especially in the realms of social networking Web sites.

\subsection{Current Efforts in Cyberbullying}

Previous work addressing cyberbullying has centered on extensive surveys unearthing the scope of the problem and on its psychological effects on victims. At a recent White House Conference on Bullying Prevention ${ }^{2}$, US President Barack Obama and First Lady Michelle Obama expressed their deep concern about this problem. The US government has created a resource bank ${ }^{3}$ that provides a survey of the current works of psychologists and educators concerning bullying, and provides resources for children, parents, teachers, and law enforcement.

Little attention, if any, to date has been devoted to technical solutions in social network software to automatically detect bullying or provide interventions directly in software interaction. Many social networks have an "Online Safety Page" that leads to resources such as the anti-bullying sites of the government or other organizations. They deal with the bullying problem primarily by people responding to explicit user complaints. Even so, in popular networks, the volume of complaints received daily quickly overwhelms the ability of small groups of complaint handlers to deal with them. The few automated detection facilities are extremely simple and ineffective, often using regular-expressions to catch a list of profane words. Solutions originally developed for spam filtering are repurposed for bullying detection [Kontostathis et al. 2010] with little precision and many false positives. In the detection section that follows, we explore the application of conventional statistical machine learning techniques for detection of bullying.

\footnotetext{
${ }^{1}$ http://www.ncpc.org/cyberbullying

${ }^{2} \mathrm{http}: / /$ www.whitehouse.gov/the-press-office/2011/03/10/background-white-house-conference-bullyingprevention

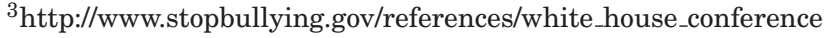


On the intervention side, little has been done directly in interactive software. Many schools provide explicit educational material to educate students about the problem and provide advice. In some US school districts such education is mandated and often takes the form of school assemblies. Typical of such educational efforts is Jay Banks's STAMP $^{4}$ which offers a set of reasonable, but vague guidelines, such as "stay away from bullies", "telling someone about a negative bullying experience", "avoiding bad situations", "making friends", and so on.

One of the most effective ways to provide education is through stories, and, among current sites, one of the best examples is MTV's The Thin Line ${ }^{5}$. This site solicits stories of possible bullying from youngsters and encourages engaging in discussion about whether a particular situation is "over the line" of acceptability. This "crowd-sourced ethics" approach is good because it respects the value of opinions of the children themselves, does not preach or assume there is a single right answer, and offers actionable advice. But the site discusses a vast variety of situations that may or may not be relevant to a particular individual or problem, and the site itself is separated from the social networks where the actual interaction takes place.

In summary, our criticism of current efforts to deal with the cyberbullying problem is that detection efforts are largely absent or extremely naïve. Intervention efforts are largely offline and fail to provide specific actionable assessment and advice.

After we provide technical descriptions of our specific efforts in the Related Work section, we will discuss specific work in natural language understanding, interactive education, and other relevant areas to particular aspects of our work.

\section{RELATED WORK}

Much of the work related to cyberbullying as a phenomenon is in the realm of social sciences and psychology. As such, this problem has not been attacked from the perspective of statistical models for detection and intervention involving reflective user interaction. Related academic work to tackle cyberbullying must be viewed from three perspectives: ethnographic studies by social scientists to gauge its prevalence, a psychological analysis of its negative impacts, and related tangential work in the NLP and the user interaction community.

\subsection{Social Sciences and Psychiatry}

A lot of research in the social sciences has been devoted to understanding the causes of cyberbullying and the extent of its prevalence, especially for children and young adults [Mishna et al. 2009]. Research in psychiatry has explored the consequences, both short and long term, that cyberbullying has on adolescents and school children and ways of addressing it for parents, educators, and mental health workers [Patchin and Hinduja 2012]. Such studies, which often involve extensive surveys and interviews, give important pointers to the scope of the problem and to designing awareness campaigns and information toolkits for schools and parents, as well as offering important algorithmic insights to parameterize detection models to catch candidate instances of cyberbullying.

\subsection{Text Categorization Tasks and Parameterization of Online Interaction Analysis}

Machine learning approaches for automated text categorization into predefined labels have witnessed a surge both in terms of applications as well as the methods

\footnotetext{
${ }^{4}$ http://jaybanks.com/anti-bullying-program/

${ }^{5} \mathrm{http}: / /$ www.athinline.org
} 


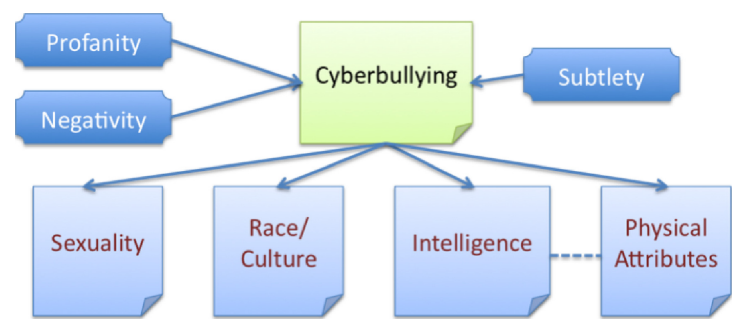

Fig. 1. Problem decomposition. A given textual comment or a post that is part of a discourse interaction on social networking Web sites is a likely candidate for cyberbullying if the underlying topic is of a sensitive nature and its has one or more contributing features of profanity, negativity, and subtlety.

themselves. Recent machine learning literature has established support-vector machines as one of the most robust methods for text categorization, used widely for email spam filters. The European Union sponsored project PRINCIP has used support vector machines using a bag-of-words approach to classify Web pages containing racist text [Greevy and Smeaton 2004]. Indeed, the support vector machine was one of our better performing methods for recognizing one of three categories of bullying remarks [Dinakar et al. 2011].

Recent work in the NLP community for classification tasks involving online interaction analysis, such as identifying fake reviews, has shown the effectiveness and importance of drawing intuitive parameters from related domains such as psycholinguistics [Ott et al. 2011]. In this work, we rely heavily on observations and intuitions from related work in the social sciences and psychology for both problem decomposition as well as feature space design.

\subsection{Similar Real-World Applications}

Apart from spam filters, applications that are of a similar nature to this work are in automatic email spam detection and automated ways of detecting fraud and vandalism in Wikipedia [Chin et al. 2010].

Very few applications have attempted to address the bullying problem directly with software-based solutions. The FearNot project [Vala et al. 2007] has explored the use of virtual learning environments to teach 8-12-year-old children coping strategies for bullying based on synthetic characters. It uses interactive storytelling with animated on-screen characters where the user gets to play one of the participants in the bullying scenario. The user may select any one of a number of response strategies to a bullying challenge, for example, fight back, run away, tell a teacher, etc. Though it provides the user with participatory education about the situations, the situations are artificially constructed. They are not part of the users' real lives. It does not make any attempt to analyze or intervene in naturally occurring situations where serious injury might be imminent and might be prevented.

\section{DETECTING BULLYING}

Cyberbullying is generally defined as the repeated injurious use of harassing language to insult or attack another individual [Ol Weus 1993]. Cyberbullying includes uploading of obscene pictures; unethical hacking into another individual's personal account on a social network, impersonation, and verbal harassment. We focus specifically on the problem of textual cyberbullying in the form of verbal harassment. Unlike spam, which is generic in the sense that it is multicast to hundreds of millions of people, bullying is aimed at a particular individual, much less often at group of individuals. We decompose cyberbullying by topics (see Figure 1). Because of the targeting of a specific 
individual, the topics involved in bullying are those of a sensitive nature that is personal to the victim. Social scientists talk extensively about the use of sensitive topics to establish a power differential between the bully and victim [Hinduja and Patchin 2008]. The set of such personal topics includes race and culture, sexuality, physical appearance, intelligence, and social rejection.

We break down the problem of topic modeling into detecting high-level contributing features, namely, profanity and contextually relevant patterns of abuse, the use of negative language devoid of profanity, as well as the employment of subtlety designed to insult another person. We experiment with statistical supervised machine learning methods to detect bullying and describe their limitations in terms of finding insulting language when there is no explicit profane or negative language. We then describe how a model of common sense reasoning can address this limitation.

This section of the article begins with a description of the problem and the corpora used for this work. Following it is a treatment of the statistical machine learning techniques used to classify text into one of the aforementioned topics. We describe how conventional machine learning techniques can detect contextually relevant patterns of abuse and the use of negative language devoid of profanity, but fail to address instances of bullying that are subtle and which need common sense reasoning for detection. We then proceed to give an overview of the Open Mind Common Sense knowledge base as well the AnalogySpace inference technique before describing a common sense reasoning model to address the difficult problem of detecting subtlety used to insult another individual.

\subsection{Rationale Behind Problem Decomposition}

When a comment or a message tends to involve sensitive topics that may be personal to an individual or a specific group of people, it deserves further scrutiny. In addition, if the same comment also has a negative connotation and contains profanity, the combination of rudeness and talking of sensitive personal topics can be extremely hurtful. Equally potent if not more so, are comments or posts that are implicitly inappropriate, that is, lacking in profanity or negativity but designed to mock or to insult.

For most children in middle school and young adults, psychological research espouses that the sensitive list of topics often assume one of the following: physical appearance, sexuality, race and culture, and intelligence [Mishna et al. 2010]. Repeated posting of such messages can lead to the victim internalizing what the bully is saying, which can be harmful to the well-being of the victim.

\subsection{Corpora}

We use two datasets for this work, YouTube and Formspring ${ }^{6}$. The YouTube dataset for experiments with statistical machine learning was obtained by scraping the social networking site www.youtube.com for comments posted on controversial (videos discussing sensitive issues such as race, culture, same-sex marriage, role of women in society, etc.) and relatively non-controversial videos (e.g., linear algebra and photoshop tutorials). Though YouTube gives the owner of a video the right to remove offensive comments from his or her video, a big chunk of viewer comments on YouTube are not moderated. Videos on controversial topics are often a rich source for objectionable and rude comments.

The comments downloaded from all the videos were arranged in random order prior to annotation. Three annotators of whom one was an educator who works with

\footnotetext{
${ }^{6} \mathrm{http}: / /$ formspring.me
} 


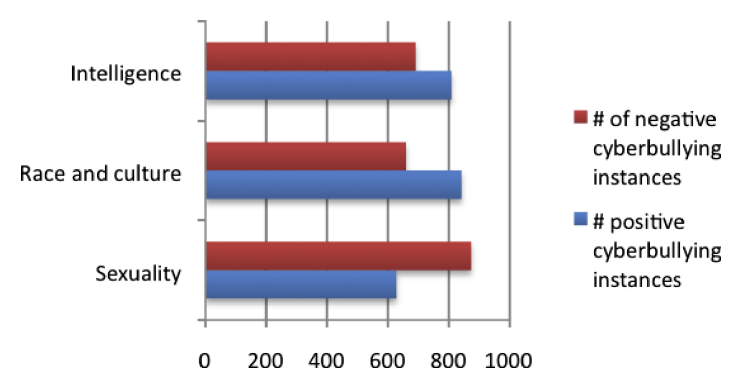

\begin{tabular}{|l|c|c|}
\hline Label/Annotation & $\begin{array}{c}\text { \# of positive } \\
\text { cyberbullying } \\
\text { instances }\end{array}$ & $\begin{array}{c}\text { \# of negative } \\
\text { cyberbullying } \\
\text { instances }\end{array}$ \\
\hline Sexuality & 627 & 873 \\
\hline Race \& Culture & 841 & 659 \\
\hline Intelligence & 809 & 691 \\
\hline
\end{tabular}

Fig. 2. The YouTube comments were annotated and grouped into three categories of 1500 instances each under sexuality, race and culture, and intelligence. 627,841 , and 809 instances were found to be positive for sexuality, race and culture, and intelligence, respectively.

middle-school children annotated each comment along the lines of three labels defined as follows.

(a) Sexuality - Negative comments involving attacks on sexual minorities and sexist attacks on women.

(b) Race and culture - Attacks bordering on racial minorities (e.g. African-American, Hispanic and Asian) and cultures (e.g. Jewish, Catholic and Asian traditions) and stereotypical mocking of cultural traditions.

(c) Intelligence - Comments attacking the intelligence and mental capacities of an individual.

After annotation, 1500 comments under each category for which the inter-rater kappa agreement was 0.4 or higher were selected for the purpose of the use of supervised learning methods (see Figure 2).

An effective strategy towards computational detection of cyberbullying must address both the explicit and direct forms of abuse, as well as the subtler, indirect ways of insulting an individual. Although the YouTube corpus is an excellent source to find direct forms of abuse involving objectionable and profane content, it lacks the personalized discourse interaction present on a more community-oriented social networking Web site. However, the YouTube corpus does serve as an excellent source for training supervised learning models to detect explicit forms of verbal abuse. The annotation task was to examine each comment and assign a label (bullying or no bullying). In the case of a positive label (bullying), the annotators were asked to assign the topic for the comment (sexuality, race and culture, or intelligence).

The MIT Media Lab partnered with the social networking Web site Formspring, whose popularity among teenagers and young adults has grown by leaps and bounds since their launch in November of 2009. From Formspring, we received a dataset of anonymized instances of bullying that were either user-flagged or caught by their moderation team. The Formspring dataset contained instances of bullying that were more targeted and specific than the YouTube corpus. It also had numerous instances of bullying involving subtlety, with use of stereotypes and social constructs to implicitly insult or malign the target. 
A ranked feature list derived from the use of supervised learning methods on the YouTube dataset was used to filter out comments from the Formspring corpus that contained blatant occurrences of profanity and negativity to obtain instances that were implicit in their intentions to bully. The same team of three annotators was asked to pick instances from this filtered dataset that pertained to topics of sexuality, namely, topics involving lesbian, gay, bisexual, and transgender (LGBT) stereotypes. The Formspring dataset contained instances that were already flagged as inappropriate by their users, allowing us to use their labels for whether an instance was cyberbullying or not.

We choose to focus the detection on the topics and stereotypes related to the LGBT community because first, bullying and cyberbullying of young adolescents based on LGBT stereotypes has been well documented both in the psychology community [Blumemenfeld and Cooper 2010] and second, the usage of LGBT stereotypes without profanity highlights the need to move beyond conventional statistical learning methods for effective detection [Heck et al. 2011]. We do not endorse any of the stereotypes through this work and seek only to use them for detection of ways of accusing or speculating about the sexuality of another individual. In the next section, we explore the use of the use of statistical supervised machine learning techniques.

\subsection{Statistical Machine Learning Techniques}

Interaction analysis on social networks is a complex phenomenon to model mathematically. The field of sociolinguistics studies interaction analysis and argues vigorously that the use of language between individuals in a social setting is parameterized by a rich set of characteristics, including identity, ascription to a particular community, personality, and affect. They argue that it is specificity and uniqueness that matter the most for effective interaction analysis. But machine learning techniques for language are often reductionist approaches that place a heavy emphasis on abstraction, generalization, and stable patterns in the data. Finding a balance between these paradigms is crucial for analyzing discourse on social networks, highlighting the importance of effective feature space design. Indeed, recent work in the computational discourse analysis community has seen the incorporation of principles from sociolinguistics for analyzing discourse [Bramsen et al. 2011].

Our approach towards using statistical supervised machine learning is to show its strengths and weaknesses in detecting cyberbullying. Since explicit verbal abuse involves the use of stereotypical slang and profanity as recurring patterns, those aspects lend themselves nicely to supervised learning algorithms. We also hypothesize that instances of cyberbullying where the abuse is more indirect and does not involve the use of profanity or stereotypical words are likely to be misclassified by supervised learning methods.

We adopt a bag-of-words supervised machine learning classification approach to identifying the sensitive theme for a given comment. We divide the YouTube corpus into $50 \%$ training, $30 \%$ validation, and $20 \%$ test data. We choose three types of supervised learning algorithms in addition to Naïve Bayes from the toolkit WEKA [Hall et al. 2009], a rule-based learner, a tree-based learner, and support-vector machines with default parameters, described briefly in the following.

- Repeated Incremental Pruning to Produce Error Reduction (JRip) is a propositional rule learner proposed by Cohen and Singer [1999]. It is a two-step process to incrementally learn rules (grow and prune) and then optimize them. This algorithm constructs a set of rules to cover all the positive instances in the dataset (those with 


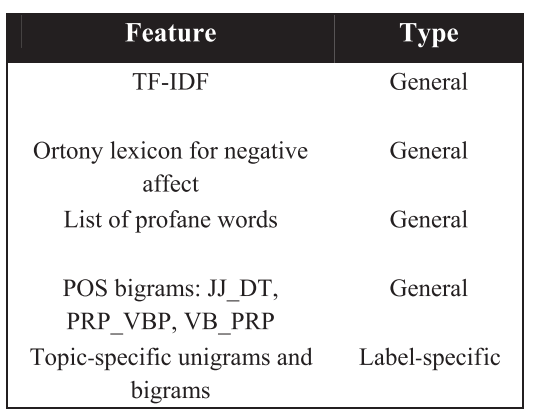

Fig. 3. Feature design. General features were common across all the datasets for both experiments. Labelspecific features consisted of unigrams that were observed in the training data.

labels \{sexuality, race and culture, intelligence\}) and has been shown to perform efficiently on large noisy datasets for the purpose of text classification [Sasaki and Kita 1998].

- J48 is a popular decision-tree-based classifier based on the C4.5 method proposed by Quinlan [1999]. It uses the property of information gain or entropy to build and split nodes of the decision tree to best represent the training data and the feature vector. Despite its high temporal complexity, J48's performance for classifying text has been shown to produce good results [Gonçalves and Quaresma 2003].

- Support-vector machines (SVM) [Cortes and Vapnik 1995] are a class of powerful methods for classification tasks, involving the construction of hyperplanes that have the largest distance to the nearest training points. Several papers reference support-vector machines as the state-of-the-art method for text classification [Gabrilovich and Markovitch 2004; Rogati and Yang 2002; Tong and Koller 2000]. We use a nonlinear poly-2 kernel [Joachims 1998] to train our classifiers, as preliminary experiments with a linear kernel did not yield statistically significant differences with a poly-2 kernel, which has also been a finding in some recent empirical evaluation of SVM kernels [Gao and Sun 2010].

In the first experiment, binary classifiers using the preceding approach were trained on each of the three datasets for each of the three labels: sexuality, race and culture, and intelligence to predict if a given instance belonged to a label or not. In the second experiment, the three datasets were combined to form a new dataset for the purpose of training a multiclass classifier using the aforementioned methods. The feature space was built in an iterative manner, using data from the validation set in increments of 50 instances to avoid the common pitfall of overfitting.

Once used, the instances from the validation set were discarded and not used again to ensure as little overfitting as possible. The trained models were washed over data from the test set for an evaluation. The kappa statistic, a measure of the reliability of a classifier, which takes into account agreement of a result by chance, was used to gauge the performance of the methods. Tenfold cross-validation was applied for training, validation, and testing for both of the experiments.

\subsection{Feature Space Design}

The feature space design for the two experiments can be categorized into two kinds: general features that are common for all three labels and specific features for the detection of each label (see Figure 3).

The intuition behind this is as follows. Negativity and profanity appear across many instances of cyberbullying, irrespective of the subject or label that can be assigned 
to an instance. Specific features can then be used to predict the label or the subject (sexuality, race and culture, and intelligence).

(a) General features. The general features consist of TF-IDF (term frequency, inversedocument frequency) weighted unigrams, the Ortony lexicon of words denoting negative connation, a list of profane words, and frequently occurring stereotypical words for each label.

(b) TF-IDF. The TF-IDF (term frequency times inverse document frequency) is a measure of the importance of a word in a document within a collection of documents, thereby taking into account the frequency of occurrence of a word in the entire corpus as a whole and within each document.

(c) Ortony Lexicon for negative affect. The Ortony lexicon [Ortony et al. 1987] (containing of a list of words in English that denotes the affect) was stripped of the positive words, thereby building a list of words denoting a negative connotation. The intuition behind adding this lexicon as unigrams into the feature set is that not every rude comment necessarily contains profanity and personal topics involving negativity are equally potent in terms of being hurtful.

(d) Part-of-speech tags. Part-of-speech tags for bigrams, namely, PRP_VBP, JJ_DT and VB_PRP were added to detect commonly occurring bigram pairs in the training data for positive examples, such "you are", "..... yourself" and so on.

(e) Label Specific Features. For each label, label-specific unigrams and bigrams were added into the feature space that was commonly observed in the training data. The label-specific unigrams and bigrams include frequently used forms of verbal abuse as well as widely used stereotypical utterances. For example, the words "fruity" and "queer" are two unigram features for the label sexuality because of their use for hurtful abuse of LGBT individuals.

We discuss an evaluation of the aforementioned supervised learning methods in Section 5. Our hypothesis is that supervised learning methods generally fare well when it comes to detecting explicit forms of verbal abuse owing to the presence of stable patterns. We anticipate in our error analysis that instances of cyberbullying that are indirect and which do not involve the use of explicit language, of which there aren't enough training samples, are likely to be misclassified by the models. In the next section we discuss the need for using common sense knowledge reasoning to detect instances of cyberbullying that could not be caught using the aforementioned conventional supervised learning methods.

\subsection{The Open Mind Common Sense Knowledge Base}

When we reason about the world, we are using our knowledge of what is expected to react to and anticipate situations. As discussed before, traditional supervised learning techniques tend to rely on explicit word associations that are present in text, but using common sense can help provide information-about people's goals and emotions and object's properties and relations-that can help disambiguate and contextualize language.

The goal of the Open Mind Common Sense (OMCS) [Singh et al. 2002] project is to provide intuition to AI systems and applications by giving them access to a broad collection of basic knowledge, along with the computational tools to work with it. This knowledge helps applications to understand the way objects relate to each other in the world, people's goals when they go about their daily lives, and the emotional content of events or situations. OMCS has been collecting common sense statements from volunteers on the Internet since 1999. At the time of this research, we have collected 
tens of millions of pieces of English language common sense data from crowd sourcing, integrating other resources, and the Semantic Web.

This knowledge allows us to understand hidden meaning implied by comments and to recognize when others are making comments designed to make us feel like our behavior is outside of the "normal". When we communicate with each other, we rely on our background knowledge to understand the meanings in conversation. This follows from the maxim of pragmatics that people avoid stating information that the speaker considers obvious to the listener [Mey 2001].

Common sense allows us a window into what the average person thinks about a concept or topic. This allows us to look for stereotypical knowledge, especially about sexuality and gender roles. OMCS knows that a girl is capable of doing housework, holding puppies, wearing bows in their hair, and babysitting and that a boy is capable of crying wolf, bagging leaves, wrestling, playing video games, and shouting loudly. More direct clues can be found in the gender associations of certain words. For example, OMCS associates dresses and cosmetics more strongly with girls. We emphasize that it is not our intention to validate or approve of any of these stereotypes, but only to use such stereotypical assertions for detection of subtle indirect forms of verbal abuse.

For the knowledge we collect to become computationally useful, it has to be transformed from natural language into more structured forms that emphasize the contextual connections between different concepts. ConceptNet represents the information in the OMCS corpus as a directed graph [Liu and Singh 2004]. The nodes of this graph are concepts, and its labeled edges are assertions of common sense that connect two concepts.

Concepts represent aspects of the world as people would talk about them in natural language, and they specifically correspond to normalized forms of selected constituents of common sense statements entered in natural language. This research uses ConceptNet 4 in which one of twenty-one different relations connects two concepts, forming an assertion. Each assertion has a notation of whether or not the relationship is considered to be negated (polarity) and a score representing the public's general opinion on whether the predicate is true or not. For example, the assertion "A skirt is a form of female attire" connects the "skirt" and "form of female attire" nodes with the "IsA" relation.

ConceptNet can also be represented as a matrix where the rows are concepts in the graph. The columns represent graph "features" or combinations of relation edges and target concepts. Features can be thought of as properties that the object might have such as "made of metal" or "used for flying". This network of concepts, connected by one of about twenty relations such as "IsA", "PartOf", or "UsedFor", are labeled as expressing positive or negative information using a polarity flag. The relations are based on the most common types of knowledge entered into the OMCS database, both through free text entry and semistructured entry. For the assertion "A beard is part of a male's face", for instance, the two concepts are "beard" and "male", the relation is "IsA", and the polarity is positive. For the assertion "People don't want to be hurt", the concepts are "person" and "hurt", the relation is "Desires", and the polarity is negative.

Each concept can then be associated with a vector in the space of possible features. The values of this vector are positive for features that produce an assertion of positive polarity when combined with that concept, negative for features that produce an assertion of negative polarity, and zero when nothing is known about the assertion formed by combining that concept with that assertion. As an example, the feature vector for "blouse" could have +1 in the position for "is part of a female attire", +1 for "is worn by girls", and -+1 for "is worn by women". These vectors together form a matrix whose rows are concepts, whose columns are features, and whose values indicate truth values of assertions. The degree of similarity between two concepts then is the dot product 
between their rows in the concept/feature matrix. This representation is discussed in detail by Havasi et al. [2009].

\subsection{The AnalogySpace Inference Technique}

In order to reason over this dataset, we needed to develop an algorithm that was both noise resistant and which took advantage of patterns inherent in how we see the world. When we determine if an object is animate, for example, we may look at the properties of that object. Does it move on its own? Is it fuzzy? Or made of metal? Is it a common pet? We also think about what objects are most similar to it. Does it look like a rabbit? Or a robot? Is it a concrete object like a pony or an immaterial quantity such as happiness?

Each question you might ask about a concept can be thought of as a "dimension" of a concept space. Then, answering a question such as where does an object lie along the "animate vs. inanimate" dimension can be thought of as reducing the dimensions of the space from every question you might ask, to just the question of interest, that is, projecting the concept onto that one dimension. We therefore use mathematical methods for dimensionality reduction, such as singular value decomposition (SVD) [Speer et al. 2008] to reduce the dimensionality of the concept-feature matrix. This determines the principal components or axes which contain the salient aspects of the knowledge, and which can be used to organize it in a multidimensional vector space. The resulting space can be used to determine the semantic similarity using linear operations over vectors representing concepts in the semantic space. Concepts close together in the space are treated as similar; these are also more likely to combine to form a valid inference.

Let us call the matrix whose rows are concepts, whose columns are features, and whose values indicate truth values of assertions as $A$. This matrix $A$ can be factored into an orthonormal matrix $U$, a diagonal matrix $\Sigma$, and an orthonormal matrix $V^{T}$ so that $A=U \Sigma V^{T}$. The singular values are ordered from largest to smallest, while the larger values correspond to the vectors in $U$ and $V$ that are more significant components of the initial A matrix. We discard all but the first $\mathrm{k}$ components-the principal components of A-resulting in the smaller matrices $U_{k}, \Sigma_{k}$, and $V_{k}^{T}$. The components that are discarded represent relatively small variations in the data, and the principal components form a good approximation to the original data. This truncated SVD represents the approximation $A A_{k}=U_{k} \Sigma_{k} V_{k}^{T}$. As AnalogySpace is an orthogonal transformation of the original concept and feature spaces, dot products in AnalogySpace approximate dot products in the original spaces. This fact can be used to compute similarity between concepts or between features in AnalogySpace.

\subsection{The Blending Knowledge Combination Technique}

While it is useful to use common sense to acquire more common sense, we benefit more when we use these techniques to learn from multiple datasets. Blending [Havasi et al. 2009] is a technique that performs inference over multiple sources of data simultaneously by taking advantage of the overlap between them. Two matrices are combined using a blending factor and then a SVD is taken over both datasets. Blending can be used to incorporate other kinds of information, such as information about stereotypes, into a common sense matrix to create a space more suited for a particular application.

We can use this technique to create a specific knowledge base to collect knowledge about different types of stereotypes beyond those in the OMCS database. Blending balances the sizes and composition of the knowledge bases such that the small size of such a knowledge base is not overpowered by the (much) larger ConceptNet. Additionally, information about implicit stereotypes may bring out other lightly stereotyped 
knowledge in the database and allows us to expand the reach of entered stereotypical knowledge. For example, adding OMCS allows us to discover that mascara, not just makeup, is usually associated with girls in the context of fashion.

Common sense can be used to fill in the gaps in other knowledge sources, both structured and unstructured or it can be designed to cover knowledge surrounding a narrow special topic. For example, in their work with SenticNet, Cambria et al. [2009] created a specialized knowledge base with information about emotions. That database has been combined with common sense and domain-specific texts to create a system that understands affect in free text [Cambria et al. 2010].

In the following sections, we build a knowledge base to perform common sense reasoning over a specific slice of cyberbullying, namely, that concerning gay and lesbian issues.

\subsection{The BullySpace Knowledge Base}

A key ingredient in tackling implicit ways of insulting another person is to transform commonly used stereotypes and social constructs into a knowledge representation. For example consider the following instance from the Formspring corpus.

put on a wig and lipstick and be who you really are

In this instance, a bully is trying to speculate about or malign the sexuality of a straight male individual implicitly, by trying to attribute characteristics of the opposite sex. (Of course, in the context of a conversation between openly gay people such a comment may be completely innocuous.) The underlying social construct here is that, in a default heterosexual context, people don't like to be attributed with characteristics of the opposite sex. This attribution is made using the common stereotype that wigs and lipstick are for women or for men who want to dress as women.

In this work, we observe the Formspring dataset and build a knowledge base about commonly used stereotypes employed to bully individuals based on their sexuality. The representation of this knowledge is in the form of an assertion, connecting two concepts with one of the twenty kinds of relations in ConceptNet. For the preceding example, the assertions added were as follows.

\begin{tabular}{|c|}
\hline lipstick is used by girls \\
lipstick is part of makeup \\
makeup is used by girls \\
a wig is used by girls \\
a toupee is used by men \\
\hline
\end{tabular}

We build a set of more than 200 assertions based on stereotypes derived from the LGBT-related instances in the Formspring database. We emphasize that our aim is not to endorse any of these stereotypes, but merely to detect their use in bullying. We then convert these assertions into a sparse matrix representation of concepts versus relations in the same manner as ConceptNet. We then use AnalogySpace's joint inference technique, blending, to merge them together to create a space that is more suited for the purpose of detecting implicit insults concerning LGBT issues. While blending, we give double post-weight to the matrix generated from the set of assertions specifically designed to capture LGBT stereotypes. Once the two matrices have been merged, we then perform an AnalogySpace inference by performing an SVD to reduce the dimensionality of the matrix by selecting only the top $k=100$ set of principal components. We now have the basic machinery required to perform common sense reasoning. 


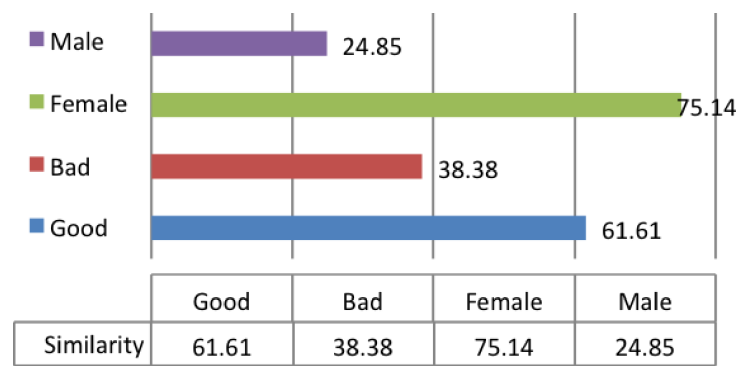

Fig. 4. Results for the comment "Did you go shopping yesterday?". Shopping as a concept is more related to females. It is also considered a good activity, in that more users in the ConceptNet database regard it as a good activity generally than bad one.

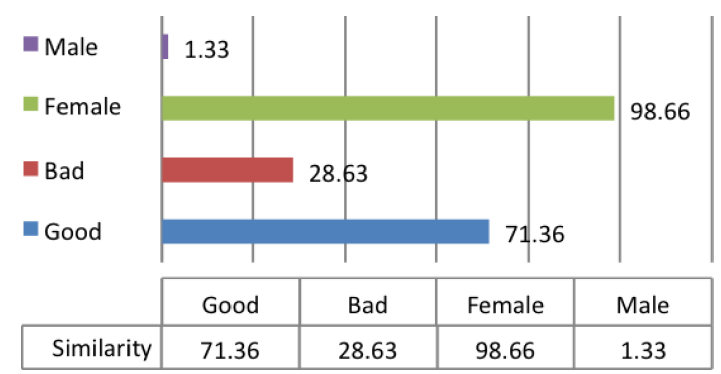

Fig. 5. The result for the comment "Hey Brendan, you look gorgeous today. What beauty salon did you visit?" is that the concept overwhelmingly tends towards a female rather than a male because of the words "gorgeous" and "beauty salon". Even though the lack of profanity or rudeness might give an impression of denoting positive affect, it relates more to females. If this comment was aimed at a boy, it might be an implicit way of accusing the boy of being effeminate, and thus a candidate sentence for cyberbullying.

\subsection{Cosine Similarity of Extracted and Canonical Concepts}

A given comment is first subjected to an NLP module to perform the standard normalization operations: removing stop-words, and tokenizing the text to have a clear separation of words from punctuation marks. Next, we extract a list of concepts from the normalized text that is also present in the concept axes of the dense matrix derived after performing the SVD as explained in the previous section.

The next task is to choose a set of canonical concepts for comparison with the concepts that have been extracted from the comment. We select four canonical concepts, namely, the affective valences positive and negative, as well as gender, namely, male and female. The idea here is to compare each extracted concept for similarity with each of the canonical concepts. This is achieved by performing a dot product over the extracted concept with a canonical concept. After this comparison, we normalize the values derived for each of the canonical concepts to get an overall measure of how similar the given comment is to each of the canonical concepts.

For example, consider the comment "Did you go shopping yesterday?". This comment is subjected to the process just described to yield similarity scores for the canonical concepts of good, bad, boy, and girl (see Figure 4).

Similarity scores derived from the preceding examples show that shopping as a concept is more oriented towards girls than boys and is largely considered as an enjoyable activity rather than a bad one. Based on these similarity scores, it can be inferred that this is a fairly innocent comment. 
Consider another comment, "Hey Brandon, you look gorgeous today. What beauty salon did you visit?". Although this contains no profanity, it does appear on the face of it, to be a comment more attributable to a girl than a boy (see Figure 5).

An analysis of the comment shows that it is overwhelmingly more similar to female concepts than male. The concepts of "gorgeous" and "beauty salons" are typically used in reference to girls rather than boys. If this comment was aimed at a boy, it might be an implicit way of accusing the boy of being effeminate, and thus becoming a candidate sentence for cyberbullying that deserves further scrutiny. Note that "gorgeous" by itself has a positive connotation, so it would be misinterpreted by merely looking for positive vs. negative words.

Here, we have focused on LGBT accusations, but in much the same way, domainspecific knowledge about other topics connected with cyberbullying such as race and culture, intelligence, and physical appearance, social rejection, etc. can be built. Canonical concepts can be selected for each of the topics in much the same way. For example, for the topic of physical appearance, the concept of 'fat' would be a canonical concept. "French fries" and "cheeseburgers," for example, would be closer to the concept of "fat" than "salads".

We discuss the evaluation of both the statistical supervised learning methods and common sense reasoning in Section 4. An error analysis on the supervised learning methods highlights the need for common sense reasoning. Of course, detection is just the first part of addressing cyberbullying. In the next part of this article, we discuss some approaches for reflective user interaction and intervention to formulate an endto-end model for tackling textual cyberbullying from detection to mitigation.

\section{INTERVENTION STATEGIES: REFLECTIVE USER INTERFACE}

\subsection{Monitoring and User Privacy}

Privacy advocates may object to having a detection algorithm scanning messages and text conversations since this is a potential violation of the user's privacy. Many common computing situations today involve the monitoring of user input. Users of the Google search engine and Gmail mail systems, for example, grant Google permission to analyze their mail or search terms in order to deliver targeted search results or targeted advertising. While many users are concerned about their privacy [Electronic Privacy Information Center 2011], others feel less concerned with having their input monitored by a program. In such cases, it is the user's responsibility to use the "optout" option to address their privacy concerns.

Minors, who have different privacy issues, are heavily engaged in the issues of cyberbullying [National Crime Prevention Council 2007]. Many parents insist on monitoring their children's social interactions, and some establish behavioral rules for the use of social networks that are extremely restrictive. For younger children, some parents resort to social networks like "Scuttle Pad"7 and "What's What?"8, which are promoted as safe networks. Similar Web sites prohibit any unmoderated commentary, any use of profanity, any social interaction with strangers, any reference to unapproved Web sites, etc. New strategies in software-based intervention will hopefully contribute to an increased feeling of safety among parents and children, while still permitting considerable freedom of expression on the child's part.

\footnotetext{
${ }^{7}$ http://www.scuttlepad.com/

${ }^{8} \mathrm{http}: / /$ whatswhat.me
} 


\subsection{Roles in the Bullying Process}

There are many roles in the cyberbullying process, including the perpetrator, the victim, and third party bystanders, such as friends, adults, moderators, and network providers. Each of these roles might elicit different kinds of reflective interfaces appropriate to their role. These roles are not mutually exclusive. Determining who is the victim and who is the perpetrator may not be an easy task. Victims may be tempted to cope with the situation by retaliating, which then thrusts them into the role of perpetrator [Stop Cyberbullying 2011]. In our collaboration with the social network provider Formspring [Formspring 2011], we learned that some negative interactions seem to start in one social network site, then spill into another. Sometimes bullies in the digital realm may be victims in the physical world. Such complexity provides a source for misinterpretation of roles and behavior. Thus directly identifying an individual of being a perpetrator or a victim may not be constructive in diminishing negative behavior. Though true bullies may never be stymied by any intervention, real or digital, however tools to support healthy digital conversations are needed.

\subsection{Reflective User Interfaces}

There are many noncomputational challenges to the reliability of algorithmic detection of cyberbullying. People may legitimately differ in what they consider bullying. Seemingly humorous responses can become unknowingly hurtful. There may be cultural differences between users which cause miscommunication. And the context of a conversation may extend beyond the social network. Given these challenges, careful consideration should be taken in planning the next actionable steps once possible candidates for potentially bullying messages can be reasonably identified.

Reflective User Interface design is a novel approach to encouraging positive digital behavioral norms. Borrowing the framework from principles espoused by Donald Schön on reflective design [Schön 1983], a Reflective User Interface is an array of solutions that might help stem or change the spread of hurtful online behavior.

Schön stated three notions of the reflective practitioner: "reflection in action," "reflection on action," and "ladders of reflections." One would reflect on behavior as it happens so as to optimize the immediately following action. One reflects after the event to review, analyze, and evaluate the situation so as to gain insight for improved practice in the future. And one's action and reflection on action makes a ladder. Every action is followed by reflection and every reflection is followed by action in a recursive manner. In this ladder, the products of reflections also become the objects for further reflections [Goel 2010].

While most of their work refers to physical products, not software interfaces, researchers, such as Sengers [2005] and Hallnäs and Redström [2001], also offer helpful insights into considering how to apply reflection to design.

Reflective User Interfaces include notifications, action delays, displaying hidden consequences, system-suggested flagging, interactive education, and the visualization of aggregated data, addressing the challenges faced by both end-users and social network moderators. Through the interface, the end-user is encouraged (not forced) to think about the meaning of a given situation, and offered an opportunity to consider their options for reacting to it in a positive way. Reflection User Interfaces resist the urge to implement heavy-handed responses, such as direct censorship. Instead, the end-user is offered options to assist them in self-adjusting or seeking external help. For the network moderator, a dashboard interface tool to display high-level network-wide overviews of aggregated negative user behavior, quickly identify problematic messages, and expedite actionable communications is in development. 


\subsection{End-User Strategies}

In providing tools to facilitate user discourse, users, moderators, and social network providers are encouraged to take an active part in determining and enforcing their own norms of healthy digital social behavior. These methods are not prescriptive, but rather options for social network providers to implement and test.

One class of interventions is notifications. Notification is drawing attention to a particular situation in order to encourage reflection. Oftentimes, people need only very subtle cues to help them understand how their behavior affects others. In faceto-face conversations in the real physical world, one has facial cues, body language, etc. to help show how one's input is being accepted. On phone calls, one can hear a person's intonation, pitch, and volume. Based on these physical responses one can quickly adapt to curb or change their behavior. In the digital realm, this is not the same especially when conversations are not in real time. Changes in the user interface could make up some of these differences [Walther et al. 2005].

Another class of interventions is interactive education. Current anti-cyberbullying efforts in schools and in youth-oriented media centers focus on educating participants about the process. Most education efforts consist of general guidelines such as warning potential perpetrators about the negative consequences to their victims and the potential damage to their own reputations. They counsel potential victims to share their experiences with friends, family, and responsible adults. They counsel potential bystanders to recognize such situations in their social circle and to take steps to defuse the situation and to provide emotional support to the participants.

While these educational efforts are positive contributions, they can be ineffective because they are disconnected from the particulars of the actual situation, both in relevance and in time and space. Guidelines are often vague and they do not address the particular details of an actual situation. Advice is usually so general that it is not directly actionable. The venue for bullying education is often school assemblies or classes, far from where bullying actually takes place.

The fact that cyberbullying occurs online presents an opportunity for intervention in real time. When a potential perpetrator is about to send a problematic message, there may be some time to encourage that person to reconsider or to give them an opportunity to rescind their message. When a potential victim is about to receive a message, there may be a few minutes to counsel them on the appropriate response or influence their immediate feelings about receiving such a message. Rather than give completely general advice, tailored advice may be offered addressing the particular kind of bullying. Such advice can be immediately actionable and can have a dramatic effect on the outcome of the situation.

\subsection{Introducing Action Delays}

A number of possible intervention techniques are aimed, not at interrupting the process, but at introducing small delays to the process in the hopes that the delay will encourage reflection. Such delays may not prevent severe cases of bullying. However major cyberbullying problems are often characterized by their rapid spread in a particular community. Slowing down the spread of negativity might in some circumstances be enough to avert a major disaster [Madlock and Westerman 2011]. The aim is to slow the spread below the "chain-reaction" rate.

Alerting the end-user that their input might be hurtful and making them wait their comment before actually submitting could also be helpful. The end-user could decide to rephrase their comment or cancel it outright. This enforces a time for Schön's "reflection in action". Generally user interface design has been focused on helping the end-user get or submit information as quickly as possible. However, there are cases 


View more comments
\begin{tabular}{|l|}
\hline Because he's a fag! ROTFL!!! \\
Wait 50 seconds to post. ${ }^{\circ *}$ X I don't want to say that.
\end{tabular}

Fig. 6. Mock-up of delay and undo operations given to the sender for a chance to reconsider message.

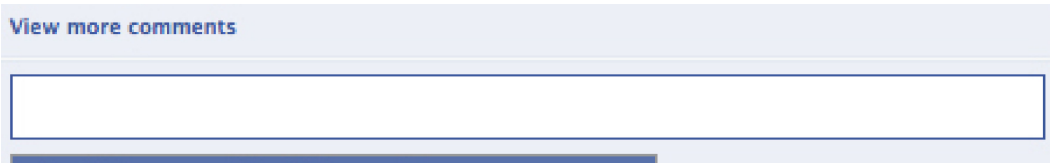

Post Comment to 770 people.

Fig. 7. Mock-up informing the sender of the consequences of sending to a large social network.

where offering the user time to reconsider and confirm that the information they are providing is truthful is warranted as in credit card purchases, applications, etc. Such enforced reflection is also common on commercial sites, which provide free services to a user. Web sites such as RapidShare, the file-sharing site ${ }^{9}$, enforce a delay so that the user has time to consider the worth of the service and the value of purchasing enhanced services or delay-free usage.

Even after making the decision to send the message, it is also helpful to provide a delay before the message is actually delivered to the recipient, giving the user the opportunity to undo the action and take the message back before it is seen by the recipient (Figure 6). Often the act of sending makes the consequences of sending seem more real to the user and triggers a "sender's remorse" response.

\subsection{Informing the User of Hidden Consequences}

Oftentimes the end-user does not realize that they are responding to the group's entire social graph, not just to the owner of the page they are commenting on. Whether a single comment or the overall tone of a thread is deemed negative by the detection algorithm, an interface change to the text label on the submit button may reflect the number of people they are communicating with.

For example, if Romeo, who has 350 friends, is posting a comment on Juliet's page, who has 420 friends, then the submit button for Romeo would reflect "Send to 770 people." (See Figure 7.)

In another example, if Tybalt's comment on Juliet's page is negative, after successfully submitting it, the system might respond with an alert box, "That's sounds harsh! Are you sure you want to send that?" If Tybalt changes his mind, an "undo" button could be made available as his comment has yet to be sent.

\subsection{Suggesting Educational Material}

For Juliet, the receiver of negative comments, the interface could provide interactive educational support. Using Google Gmail ad-like text messages could be placed next to the negative comments offering the user support: "Wow! That sounds nasty! Click here for help." The small text message links to external Web sites related to supporting victims of bullying. This also provides an easy conduit for external support agencies to connect their materials to the individuals for whom they work. Social network

\footnotetext{
${ }^{9} \mathrm{http} / / /$ rapidshave.com
} 


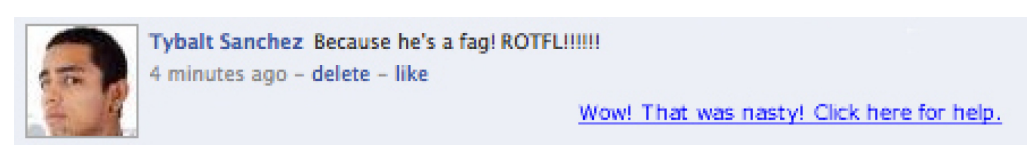

Fig. 8. Mock-up of a small text message offering educational material to users after detection of a problematic message.

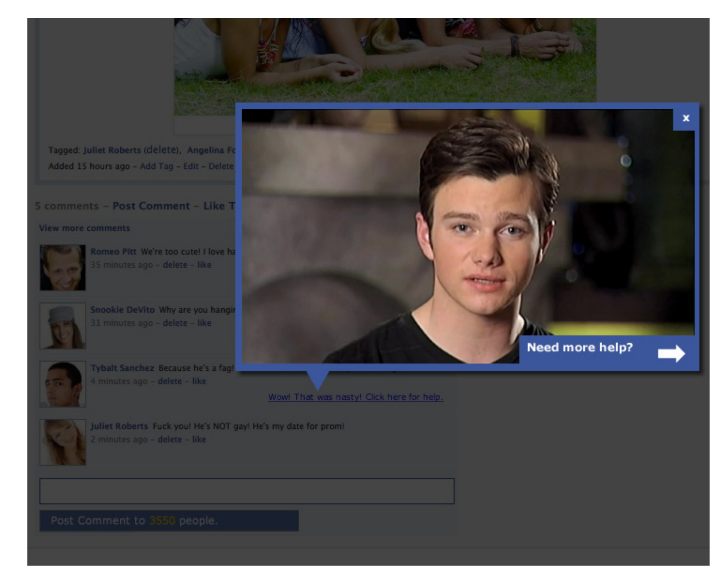

Fig. 9. Mock-up of an anti-bullying video from http://www.itgestbetter.org is presented after the user clicks on a help message link.

providers could partner with outside social agencies to craft appropriate material to link to and utilize this method of helping their end-users (Figure 8).

Educational materials created to support victims of bullying are often too general. And the actual support provided to victims usually happens long after the bullying event. Even more ambitious than a link to external content, we are building an interface strategy to provide short targeted video suggestions of what to do next or how to respond. The detection algorithm could analyze the user's comments and situation, and then align it to preselected stories or educational materials representing similar issues.

The small text link is the first point of entry for the user. The interface is developed to include functionality similar to an expert help system [Ignizio 1991]. Designing an intelligent help system to best serve the end-user is complex and difficult as discussed by Molich and Nielsen [1990]. The text link initiates the end-user "reflection on action." After the educational material is presented, the interface could ask the user whether or not the story provided was in fact a good match and if it was useful. If the user requests more help, suggested solutions and materials are provided in a tiered manner. The help support system would record the user interactions, so if the user requests more help in the future, the system knows it has provided assistance before and would not treat the interaction as a new occurrence. By allowing the user to opt in or out at any stage of engagement, the support would become contextual, prescriptive, and desired rather than overbearing and obstructive (Figure 9).

\subsection{Social Network Provider Strategies}

The primary concern of social network providers and group moderators is to provide a safe and welcoming environment for their community. It is not necessary to detect or control every single incident of bullying that occurs. Most important is to prevent an initially trivial incident from escalating or spreading within a community and 
becoming the social norm. An important part of maintaining quality social networks would include giving moderators an aggregate view of the behavior of their end-users. Patterns of escalation or spreading of bullying caught early give moderators opportunities to intervene before serious problems arise.

\subsection{Flagging of Messages}

Many social networks allow end-users to flag messages as inappropriate. Human moderators, who use their judgment to decide whether action is warranted, often review such flagged messages. Automated detection algorithms can contribute to this flagging process by suggesting to a participant that a message might need to be flagged. It can also help by prioritizing a list of flagged messages for review by the human moderator. This prioritization is essential, because the volume of flagged messages in large-scale social networks can overwhelm moderators.

Flagged comments can be displayed in various ways. The comment can be visibly marked or hidden from the public and particular end-users or made available for viewing to only the receiver and sender of the comment. The moderator could also hold a flagged comment for review.

\subsection{Visualization of Online Bullying}

A dashboard interface for moderators and social network providers to visualize highlevel overviews of their network's end-user behavior is in development. One view of the dashboard could serve some of the same functions as back channels [McNely 2009] in calling attention to possibly problematic situations. A social network using a detection algorithm may not want to make any changes to the end-user interface until there is an understanding of the scope and domains of their end-users' negative behavior.

The dashboard would have many display views to reflect key semantic terms, social clusters, events, and basic demographics derived from the network social graph. It could also display the actual flagged messages related to the semantic terms prioritized in order of seriousness by the detection algorithm. Providers could use the dashboard to help their moderators get an overview of their supervised space on the network.

Problems in the real world are often reflected in the digital world [Hinduja and Patchin 2007]. As a courtesy service, the social network provider could also provide third parties, such as police and school administrators/staff, a version of the dashboard using sanitized (anonymous) user names. In this scenario, a school administrator would be able to see an overview of the digital behavior of their school's student population. For example, without giving actual screen names and real conversations, the school could find out that there are problems such as gay bashing during the weeks leading up to the prom. This information could be vital in scheduling appropriate realworld intervention strategies at the school.

Due to the public's growing hypersensitivity of cyberbullying, sometimes one publicized incident can overrepresent the severity of problems in a social network [Collier 2011]. A public version of the dashboard could provide transparency and a more balanced overview about the network's problems based on objective data (see Figure 10).

\section{EVALUATION AND DISCUSSION}

The statistical models discussed in Section 3 were evaluated against 200 unseen instances for each classifier. The labels assigned by the models were compared against the labels that were assigned to the instances during annotation. The accuracy and kappa values of the classifiers are in Figures 11 and 12. 


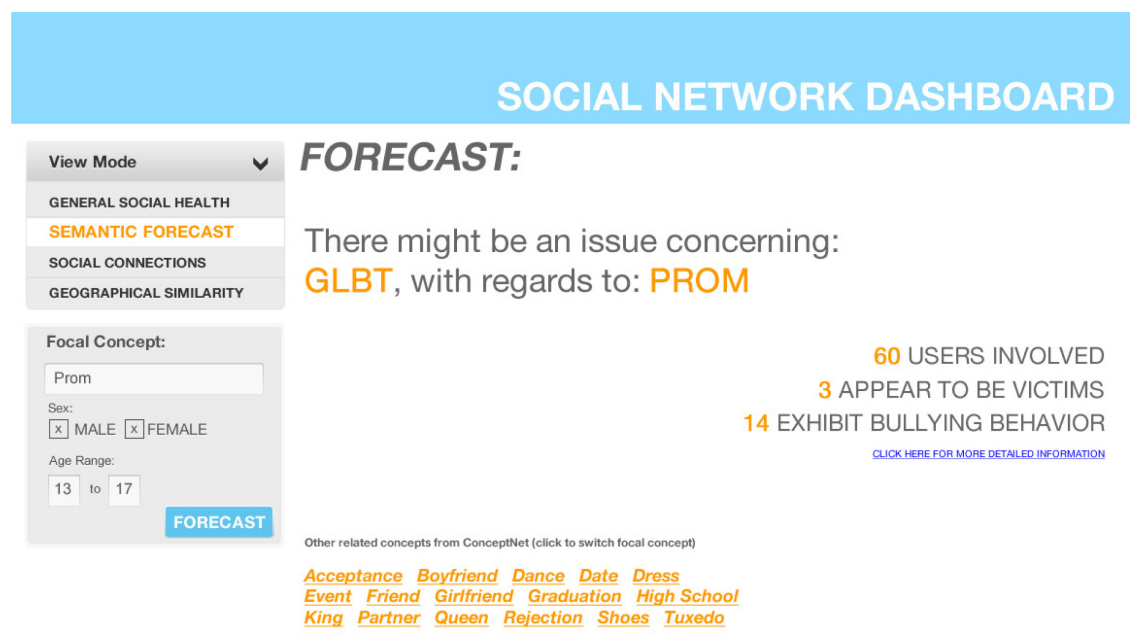

Fig. 10. Mock-up of social network dashboard displaying the community visualization environment.

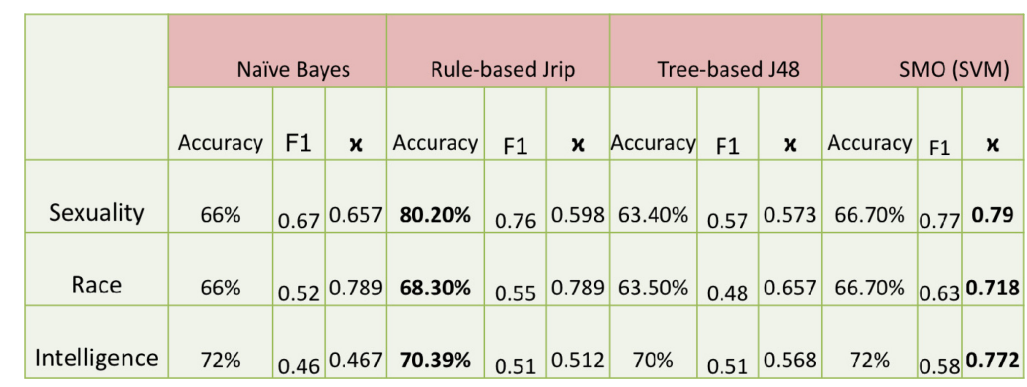

Fig. 11. Binary classifiers for individual labels.

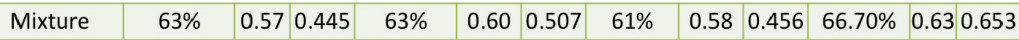

Fig. 12. Multiclass classifiers for the merged dataset. Binary classifiers trained for individual labels fare better than multiclass classifiers trained for all the labels. JRip gives the best performance in terms of accuracy, whereas SMO is the most reliable as measured by the kappa statistic.

To avoid lexical overlap, the 200 instances for each label were derived from video comments that were not part of the original training and validation data. Prior work on the assessment of classifiers suggests that accuracy alone is an insufficient metric to gauge reliability. The kappa statistic $\varkappa$ (Cohen's kappa), which takes into account agreement by chance, has been argued as a more reliable metric in conjunction with accuracy [Carletta 1996]. We evaluate each classifier in terms of the accuracy, the F1-score, as well the kappa statistic.

Multiclass classifiers underperformed compared to binary classifiers. In terms of accuracy, JRip was the best, although the kappa values were best with SVM. SVM's high kappa values suggest better reliability for all labels. Naïve Bayes classifiers for all labels perform much better than J48.

\subsection{Error Analysis of the Supervised Learning Models}

As we hypothesized, an error analysis on the results reveals that instances of bullying that are apparent and blatant are simple to model because of their stable, repetitive 
patterns. Such instances either contain commonly used forms of abuse or profanity or expressions denoting a negative tone. For example, consider the following instances.

u1 as long as fags don't bother me let them do what they want

$\mathrm{u} 2$ hey we didn't kill all of them, some are still alive today. And at least we didn't enslave them like we did the monkeys, because that would have been more humiliating

Both of these instances shown above (the first pertaining to sexuality and the second pertaining to race) contain unigrams and expressions that lend them to be positively classified by the models. Instances such as the ones shown, which contain lexical and syntactic patterns of abuse, lend themselves to supervised learning for effective detection. However, the learning models misclassified instances that do not contain these patterns and those that require at least some semantic reasoning. For example, consider the following instances.

u3 they make beautiful girls, especially the one in the green top

u4 she will be good at pressing my shirt

In the first instance, which was posted on a video of a middle-school skit by a group of boys, the bully is trying to ascribe female characteristics to a male individual. The instance has no negativity or profanity, but implicitly tries to insult the victim by speculating about his sexual orientation. "Tops" and "beautiful" are concepts that are more associated with girls than boys, and hence if attributed to the wrong gender, can be very hurtful. In the second instance, a bully exploits the common sexist stereotype that pressing clothes is an act reserved primarily for women. The learning models misclassified these two instances, as it would need to have some background knowledge about the stereotypes and social constructs and reason with it. In the next section, we discuss our work with supervised learning models in the context of related approaches to sentiment analysis.

5.1.1. Discussion. Prior research in sentiment analysis has focused on sentiment polarity for opinion analysis for movie and product reviews [Pang and Lee 2008]. However, the nature of interpersonal and group interaction on social networks is different from sentiment polarity of reviews from two perspectives, and hence it is difficult to compare them. First, interaction of social networks (like Formspring) as a sociolinguistic phenomenon is more targeted towards a specific audience (an individual or a group of individuals), while movie and product reviews are intended for a larger, more general audience. Second an analysis of discourse on social networks involves deeper attributes such as identity, ascription to a particular community, personality and affect, which is more than just sentiment polarity of movies or product reviews where there is a prior acknowledgement of the domain under scrutiny.

Recent work with affect recognition in text has attempted a fine-grain compositional approach to gauging emotions [Neviarouskaya et al. 2009]. While we did not adopt a finer granularity approach towards gauging emotion, we emphasize that our focus was on overcoming the limitations of supervised learning methods in catching indirect, subtle forms of abuses using social constructs which require reasoning along relevant dimensions (such as gender roles). In the next section, we discuss the evaluation of the experiments involving common sense reasoning. 


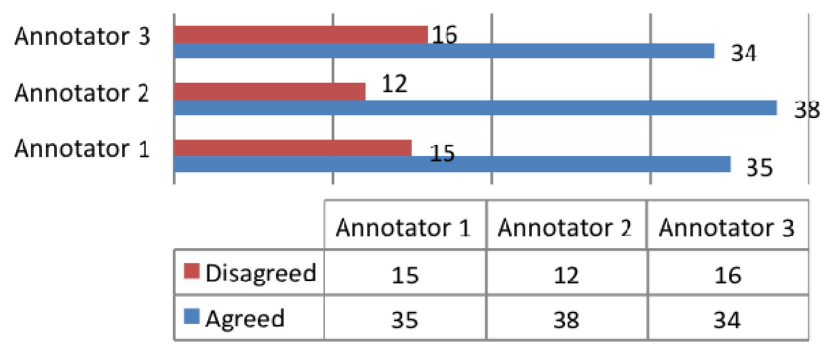

Fig. 13. Evaluation of the common sense reasoning model. Fifty instances from the Formspring dataset were evaluated by the model to generate similarity scores for the canonical concepts "girl" and "boy". The same three annotators validated the results. All 50 instances were previously flagged as instances of cyberbullying.

\subsection{Evaluation of Common Sense Reasoning Models}

For an evaluation of the detection element of common sense reasoning, it is essential to have a dataset that contains instances of cyberbullying devoid of profanity and implicitly crafted to insult or malign a user. To address the specific slice of LGBT bullying in this work, it is essential to have a test dataset that pertains to LGBT bullying as well as some instances that do not pertain to LGBT issues.

We build such a test by performing a filtering operation on the original Formspring dataset as follows. The same set of people who annotated the YouTube corpus were asked to pick instances from the Formspring dataset that satisfied the dual criteria of not having any profanity and implicitly trying to attack, insult, or speculate on the sexuality of the victim. Of the 61 instances of bullying that were obtained from the three annotators, 50 instances were made into a test set. It is important to keep in mind that the original Formspring corpus contains instances that have already been flagged as bullying. Hence the annotators were not asked to check if an instance was bullying or not.

Since the goal of the detection approach that we take in this article is to prioritize reported instances of bullying based on similarity scores, we adopt a similar approach for this test dataset. The test dataset was evaluated with the approach mentioned in Section 2 to generate similarity metrics for each instance with the canonical concepts "girl" and "boy". The results were shown to each of the three annotators to check if they agreed with the metrics generated by the common sense reasoning model. The results are shown in Figure 13.

5.2.1. Error Analysis of the Common Sense Reasoning Model. An analysis of the instances for which the annotators disagreed with the common sense reasoning model can be classified into three kinds. The first kind were instances in which the similarity metrics did not make common sense, and the second kind were instances in which the annotators did not agree on the scale of the similarity metrics.

Most of the instances for which similarity metrics did not make common sense were largely due to sparsity of data in the space that was built for performing the SVD. For instance, consider the following example with similarity metrics that did not make common sense.

George Michael or Elton John?

This instance received an extremely high score for the concept "boy" due to the names of the individuals mentioned. However, a deeper analysis shows that the 
individuals are celebrity singers who also have one thing in common: they are both openly gay. The three annotators all agreed that by suggesting that an individual likes these singers, the perpetrator is implicitly trying to speculate or mock their sexuality. To address such instances, one really needs to have more canonical concepts than "girl" and "boy".

Those instances for which the relative scale of the similarity scores was not agreeable to the annotators can be attributed to the scoring process in ConceptNet, which relies on the frequency of an assertion to determine its relative scoring. For example, consider the following instance.

why did you stop wearing makeup?

This example generated normalized similarity scores as follows: $60.7 \%$ for the concept of girl and $32.2 \%$ for the concept of boy. While there are men, such as actors who routinely wear makeup, makeup is more strongly associated with women than men. This suggests the need for an in-context weighting of assertions. Makeup and costumes, for example are more likely to be associated with individuals in the performing arts, irrespective of their gender.

It is clear from the evaluation that the problem of sparsity as well as the ability to individually weight an assertion will be vital if this approach is to be implemented in a large-scale user community. One can imagine crowd-sourced collection of such relevant social constructs as common sense assertions from both users and moderators of social networks.

\subsection{Reflective User Interface Evaluation}

The Reflective User Interface strategy of suggesting educational material, as discussed in Section 4.7, was evaluated in a small user study, testing the differences between dynamic in-context targeted advice in the user interface, targeted static advice in the user interface, and the typical "help" link user interaction found on most social networks. The study included five participants, consistent with the findings Nielsen suggests when conducting a user test. [Nielsen 2000] A fully functioning hypothetical social network, called Fakebook, was built as this platform for testing both detection algorithms and user interface strategies. Fakebook was graphically styled and patterned after the popular social network Facebook so that users would feel familiar with its interface (see Figure 14).

Prior to the user study, a mock conversation between three imaginary persons was staged to present a bullying interaction. While the dialogue was fictional, it modeled real messages found from research data provided by MTV's A Thin Line Web site ${ }^{10}$. Using the detection classifier, each message was scanned for being a possible case of bullying.

Three different versions of a wall (an interface that allows users to send and receive messages) [Wall 2011] conversation were created, each varying the type of advice offered by the user interface. In the first version, a small text link stating, "Click here for help," was placed next to the messages positively identified by the classifier as being a candidate for bullying. Once clicked, the link would bring up a modal window (pop-up window) containing a short paragraph of advice for coping with bullying situations (Figure 15). While the advice was hand-curated from a Web site specializing in

${ }^{10} \mathrm{http}: / / \mathrm{www}$. athinline.org 

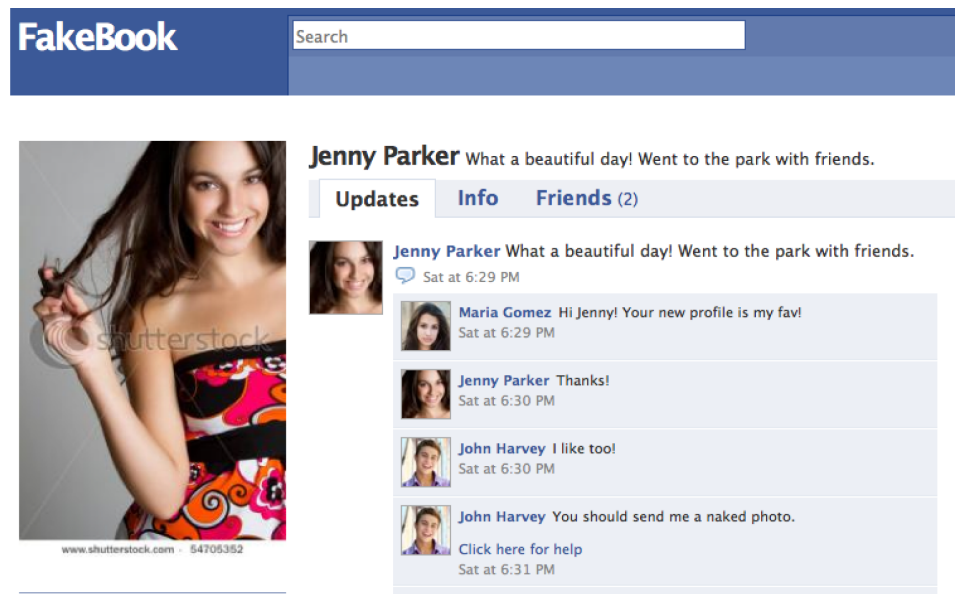

Jenny Parker What a beautiful day! Went to the park with friends.

Updates Info Friends (2)

(2)

Jenny Parker What a beautiful day! Went to the park with friends.

Sat at 6:29 PM

A Maria Gomez Hi Jenny! Your new profile is my fav!

2. Maria Gomez $6: 29 \mathrm{PM}$

(2) Jenny Parker Thanks!

D. John Harvey I like tool

2. John Harvey

7 J. John Harvey You should send me a naked photo.

1. Click here for help
Sat at 6:31 pM

2. Jenny Parker Go away!

Member Type: Regular Member

Profile Views: 17 views

Friends: 2 friends

Last Update: Sat at 6:29 PM

Joined: Sat at 4:28 PM

1. John Harvey Please!

(2) John Harvey 1 just sent you a photo of my dick.

1. Click here for help Sat at 6:33 PM

DIJ Jenny Parker Stop with the sext!

Click here for help

Sat at 6:34 PM

18 John Harvey I saw your breasts on Tom's page. Why not?

21. Click here for help
Sat at 6:35 PM

DJ Jenny Parker Enough! Go away!
Sat at 6:35 PM

$\sqrt{2}$ John Harvey whatever you bitch! Die a virgin for all I care

Click here for help

Fig. 14. Fakebook, the fully functioning social network built as a testing platform. The "Wall" interface is shown with in-context links to targeted help.

cyberbullying coping advice for both children and parents ${ }^{11}$, it is dynamically displayed based on the detection algorithm's analysis of the bullying message. Jakob Nielsen's Ten Usability Heuristics provides a relevant guide for the modal window interface design decision. [Nielsen 2011] "Recognition rather than recall" suggests that, "the user should not have to remember information from one part of the dialogue to another." The interface presents the help advice in the same viewing area as the potentially negative interaction.

The second version of the interface looked exactly as the first, but the content of the "Click here for help" modal window consisted of a single web link to a Web site [Stop Cyberbullying 2011]. The suggested Web site represents many similar Web sites that are listed in the help sections of many social networks. These links, while helpful, are often hard for end-users to find. And they are located on Web pages separate from the user interaction space. The third version of the interface contained no targeted advice links. In every version, the standard help link was present. Clicking this link brought users to a page mirroring the current Facebook help page (Figure 16).

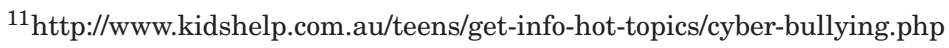




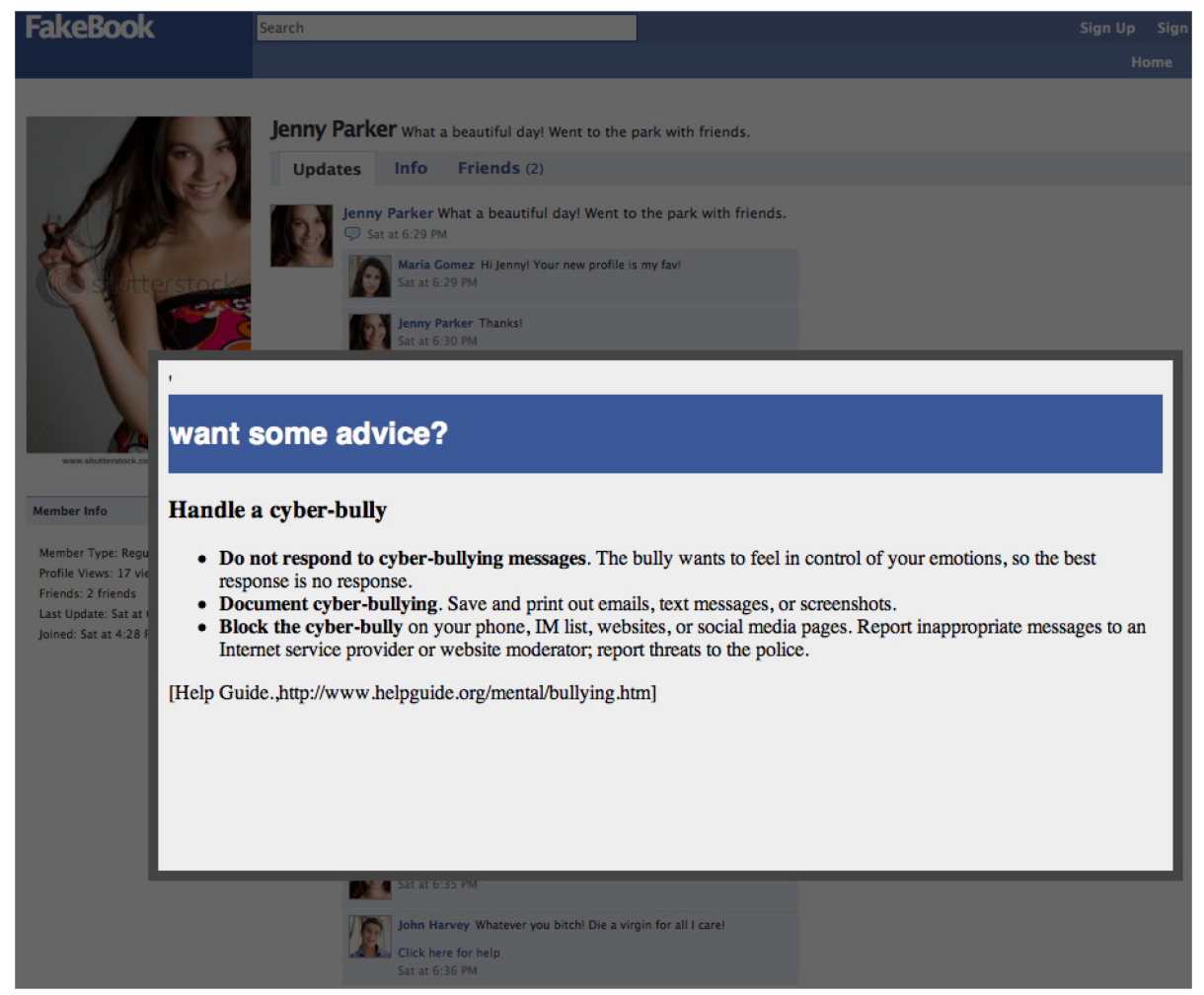

Fig. 15. After the end-user clicks on a link for more help, the Fakebook modal window displays in-context targeted help.

For the user study, participants used the Fakebook social network to take a survey. They were shown the three versions of the wall of Jenny, a fictional character, and her conversation with two people, John and Maria. Participants were asked to read through the conversational thread, imagining themselves as each of one of the characters, and then ask questions. The five participants were asked to click on each "Click here for help" link while reading through the conversation. Participants were told that Jenny was the victim, John was the bully, and Maria was a third-party bystander.

The survey used Likert scale questions, answering with strongly disagree, disagree, neither agree nor disagree, agree, or strongly agree. Participants were asked the same three questions for each version of the wall interface. The first question was, "Imagine you are Jenny. Assuming Jenny is the victim, when I clicked on the advice links I considered the advice helpful in the situation." The second question, "Imagine you are John. Assuming John is the bully, when I clicked on the help links, I felt reflective about my behavior and how it might have affected Jenny." And the third question, "Imagine you are Maria. Assuming the Maria is a bystander, when I clicked on the links, I reflected on how the messages might have affected Jenny." (See Tables I-IV.)

We were encouraged that participants overwhelming preferred the interface with targeted in-context advice, which concurs with our assertion that targeted help within the user interface at the point of the bullying interaction would be more helpful to the end-user than the typical "help" link support provided by social networks. There are 


\begin{tabular}{l}
\hline Basics \\
\hline Trouble Using Facebook \\
\hline $\begin{array}{l}\text { Report Abuse or Policy } \\
\text { Violations }\end{array}$ \\
\hline $\begin{array}{l}\text { Ads and Business } \\
\text { Solutions }\end{array}$ \\
\hline Safety Center \\
\hline Community Forum \\
\hline
\end{tabular}

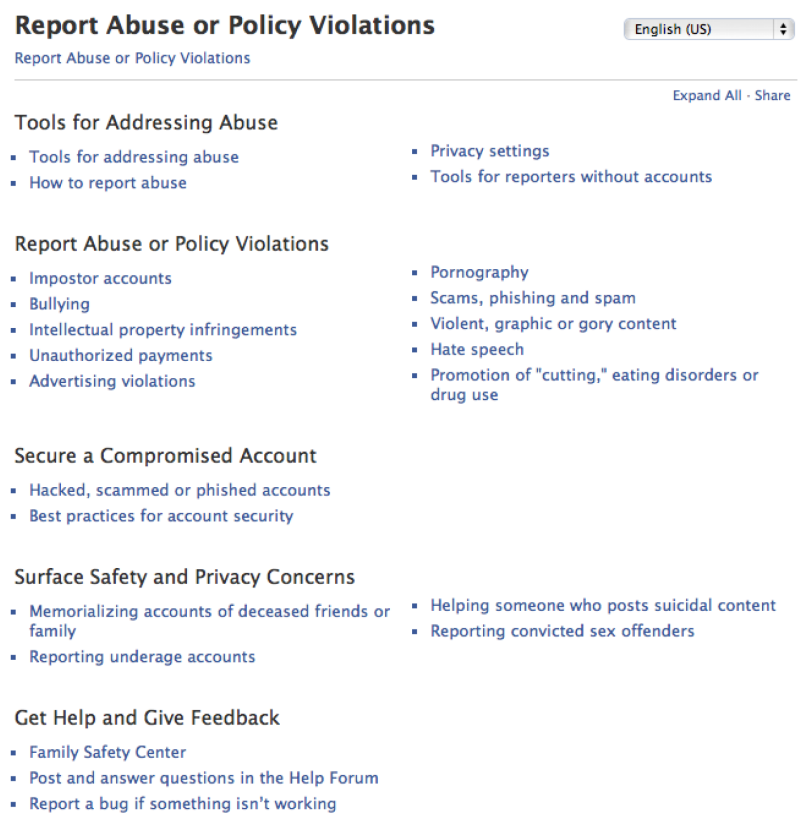

Fig. 16. Facebook's help page.

Table I. The Study Protocol

\begin{tabular}{|l|l|l|l|}
\hline & \multicolumn{1}{|c|}{$\begin{array}{c}\text { Interface 1: } \\
\text { In-Context Dynamic Help }\end{array}$} & \multicolumn{1}{c|}{$\begin{array}{c}\text { Interface 2: } \\
\text { (Control) Static Help }\end{array}$} & $\begin{array}{c}\text { Interface 3: } \\
\text { No Interface Change }\end{array}$ \\
\hline Motivations & $\begin{array}{l}\text { Targeted help is more } \\
\text { appropriate to the } \\
\text { situation of the end-user. } \\
\text { By providing the help } \\
\text { in the same interface as } \\
\text { the bullying interaction, } \\
\text { the advice becomes } \\
\text { actionable. }\end{array}$ & $\begin{array}{l}\text { Static help provided to the } \\
\text { end-user in the same } \\
\text { interface as the bullying } \\
\text { interaction, would have } \\
\text { more perceived value to } \\
\text { the end-user than no } \\
\text { in-interface assistance } \\
\text { at all. }\end{array}$ & $\begin{array}{l}\text { This represents the } \\
\text { standard social network } \\
\text { interface. }\end{array}$ \\
\hline User Protocol & $\begin{array}{l}\text { Participants were asked } \\
\text { to click help links. }\end{array}$ & $\begin{array}{l}\text { Participants were asked to } \\
\text { click help links. }\end{array}$ & $\begin{array}{l}\text { Participants were asked } \\
\text { to click the standard } \\
\text { "help" link. }\end{array}$ \\
\hline
\end{tabular}

few, if any, social networks providing in-context dynamic support for cyberbullying. As a result, there are few intervention models to employ in the manner we propose. The second interface providing static help within the user interaction could serve as intermediate step towards providing end-users with support.

\section{CONCLUSION}

Cyberbullying of youth on social networks is a growing problem, as recent news stories detailing suicides of bullied teenagers attest. In less extreme forms, this problem is widespread with a significant fraction of young people bullying incidents happening to them or to their social circle. Such incidents threaten the continued growth of online social networks for young people. This would be a shame, because social networks provide many benefits for youngsters, including opportunities for forming new 
Table II. Results for Interface 1 Using In-Context, Targeted Dynamic Help

\begin{tabular}{|c|c|c|c|c|c|}
\hline \multicolumn{6}{|c|}{ Interface 1: In-Context Dynamic Targeted Help } \\
\hline & $\begin{array}{l}\text { Strongly } \\
\text { Disagree }\end{array}$ & Disagree & $\begin{array}{c}\text { Neither Agree } \\
\text { nor Disagree }\end{array}$ & Agree & $\begin{array}{l}\text { Strongly } \\
\text { Agree }\end{array}$ \\
\hline $\begin{array}{l}\text { Imagine you are Jenny. } \\
\text { Assuming Jenny is the } \\
\text { victim, when I clicked on } \\
\text { the advice links I considered } \\
\text { the advice helpful in the } \\
\text { situation. }\end{array}$ & $0 \%$ & $0 \%$ & $0 \%$ & $20 \%$ & $80 \%$ \\
\hline $\begin{array}{l}\text { Imagine you are John. } \\
\text { Assuming John is the bully, } \\
\text { when I clicked on the help } \\
\text { links, I felt reflective about } \\
\text { my behavior and how it } \\
\text { might have affected Jenny. }\end{array}$ & $0 \%$ & $20 \%$ & $0 \%$ & $40 \%$ & $40 \%$ \\
\hline $\begin{array}{l}\text { Imagine you are Maria. } \\
\text { Assuming the Maria is a } \\
\text { bystander, when I clicked } \\
\text { on the links, I reflected on } \\
\text { how the messages might } \\
\text { have affected Jenny. }\end{array}$ & $0 \%$ & $0 \%$ & $0 \%$ & $20 \%$ & $80 \%$ \\
\hline
\end{tabular}

Table III. Results for Interface 2 Using Static Help

\begin{tabular}{|c|c|c|c|c|c|}
\hline \multicolumn{6}{|c|}{ Interface 2: (Control) Static Help } \\
\hline & $\begin{array}{l}\text { Strongly } \\
\text { Disagree }\end{array}$ & Disagree & $\begin{array}{c}\text { Neither Agree } \\
\text { nor Disagree }\end{array}$ & Agree & $\begin{array}{l}\text { Strongly } \\
\text { Agree }\end{array}$ \\
\hline $\begin{array}{l}\text { Imagine you are Jenny. } \\
\text { Assuming Jenny is the } \\
\text { victim, when I clicked on } \\
\text { the advice links I considered } \\
\text { the advice helpful in the } \\
\text { situation. }\end{array}$ & $0 \%$ & $40 \%$ & $40 \%$ & $20 \%$ & $0 \%$ \\
\hline $\begin{array}{l}\text { Imagine you are John. } \\
\text { Assuming John is the bully, } \\
\text { when I clicked on the help } \\
\text { links, I felt reflective about } \\
\text { my behavior and how it } \\
\text { might have affected Jenny. }\end{array}$ & $20 \%$ & $60 \%$ & $20 \%$ & $0 \%$ & $0 \%$ \\
\hline $\begin{array}{l}\text { Imagine you are Maria. } \\
\text { Assuming the Maria is a } \\
\text { bystander, when I clicked } \\
\text { on the links, I reflected on } \\
\text { how the messages might } \\
\text { have affected Jenny. }\end{array}$ & $0 \%$ & $80 \%$ & $20 \%$ & $0 \%$ & $0 \%$ \\
\hline
\end{tabular}

relationships, strengthening existing ones, sharing interests, and practicing reading and writing in a personally meaningful context.

In addition to educational efforts in schools, increasing awareness, and discussion of the problem between adults and young people, we believe technical solutions with social network software will form an important component of identifying and reducing the harm associated with this problem. We have presented a suite of capabilities for social network software to address detection of potentially bullying messages and to intervene by notifying participants and network moderators, managing message access, and offering targeted educational material. 
Table IV. Results for Interface 3, Which Contained No Interface Changes

\begin{tabular}{|c|c|c|c|c|c|}
\hline \multicolumn{6}{|c|}{ Interface 3: No Interface Changes } \\
\hline & $\begin{array}{l}\text { Strongly } \\
\text { Disagree }\end{array}$ & Disagree & $\begin{array}{c}\text { Neither Agree } \\
\text { nor Disagree }\end{array}$ & Agree & $\begin{array}{l}\text { Strongly } \\
\text { Agree }\end{array}$ \\
\hline $\begin{array}{l}\text { Imagine you are Jenny. } \\
\text { Assuming Jenny is the } \\
\text { victim, when I clicked on } \\
\text { the advice links I considered } \\
\text { the advice helpful in the } \\
\text { situation. }\end{array}$ & $100 \%$ & $0 \%$ & $0 \%$ & $0 \%$ & $0 \%$ \\
\hline $\begin{array}{l}\text { Imagine you are John. } \\
\text { Assuming John is the bully, } \\
\text { when I clicked on the help } \\
\text { links, I felt reflective about } \\
\text { my behavior and how it } \\
\text { might have affected Jenny. }\end{array}$ & $100 \%$ & $0 \%$ & $0 \%$ & $0 \%$ & $0 \%$ \\
\hline $\begin{array}{l}\text { Imagine you are Maria. } \\
\text { Assuming the Maria is a } \\
\text { bystander, when I clicked } \\
\text { on the links, I reflected on } \\
\text { how the messages might } \\
\text { have affected Jenny. }\end{array}$ & $100 \%$ & $0 \%$ & $0 \%$ & $0 \%$ & $0 \%$ \\
\hline
\end{tabular}

Fully general natural language understanding still remains beyond reach. But we have shown that state-of-the-art natural language processing, augmented by a common sense reasoning technique, specialized knowledge bases concerning bullying, and a novel reasoning technique can result in accuracies approaching $80 \%$ in identifying potentially bullying messages with significant agreement ratings with human labelers.

We have also presented a wide range of potential intervention techniques, ranging from subtle changes to the messaging interface to personalized, interactive educational material and "air traffic control" overviews to help network providers maintain positive social norms. These interfaces show how adding intelligence to an interactive interface in cooperation with the various roles that human users play can make social network applications more effective in their goal of enhancing human relationships.

\section{REFERENCES}

Blumemenfeld, W. J. And Cooper, R. M. 2010. LGBT and allied youth responses to cyberbulling: Policy implication. Int. J. Critical Pedagogy.

Boyd, D. 2007. Why Youth (Heart) Social Network Sites: The Role of Networked Publics in Teenage Social Life. MacArthur Foundation Series on Digital Learning, Youth, Identity, and Digital Media, David Buckingham, Ed., MIT Press, Cambridge, MA.

Bramsen, P., Escobar-Molano, M., Patel, A., And Alonso, M. 2011. Extracting social power relationships from natural language. In Proceedings of the 49th Annual Meeting of the Association for Computational Linguistics: Human Language. 773-782.

CAmbria, E., Hussain, A., HAVAsi, C., AND ECKL, C. 2009. AffectiveSpace: Blending common sense and affective knowledge to perform emotive reasoning. In Proceedings of the Workshop on Opinion Mining and Sentiment Analysis.

CAmbria, E.,SPeER, R., AND HaVAsi, C. 2010. SenticNet: A publicly available semantic resource for opinionmMining. In Proceedings of the AAAI Symposium on Common Sense Knowledge.

CARlettA, J. 1996. Assessing agreement on classification tasks: The kappa statistic. Computat. Linguis. $22,2,249-254$.

Chin, S., Street, N., Srinivasan, P., and Eichmann, D. 2010. Detecting Wikipedia vandalism with active learning and statistical language models. In Proceedings of the 4th Workshop on Information Credibility. 3-10.

Cohen, W. And Singer, Y. 1999. A simple, fast, and effective rule learner. In Proceedings of the 16th National Conference on Artificial Intelligence. 
Collier, A. 2011. A cyberbullying epidemic? No! Web log comment. http://www.netfamilynews.org/?p=30592.

Cortes, C. AND VAPNIK, V. 1995. Support-vector networks. Machine Learn. 20. http://www.springerlink.com/content/k238jx04hm87j80g/.

DinAKAR, K., REICHART, R., AND Lieberman, H. 2011. Modeling the detection of textual cyberbullying, In Proceedings of the International Conference on Weblog and Social Media (Social Mobile Web Workshop).

Electronic Privacy Information Center. 2011. Google Privacy FAQ. http://epic.org/privacy/gmail/faq.html.

GABRILOVICH, E. AND MARKOVITCH, S. 2004. Text categorization with many redundant features: Using aggressive feature selection to make SVMs competitive with C4.5. In Proceedings of the 21st International Conference on Machine Learning. ACM, New York.

GAO, Y. AND SUN, S. 2010. An empirical evaluation of linear and nonlinear kernels for text classification using support vector machines. In Proceedings of the 7th International Conference on Fuzzy Systems and Knowledge Discovery. 1502-1505.

Goel, S. 2010. Design is a reflective practice: A summary of Schon's views. Engineering \& Computing Education: Reflectins and Ideation. Web post. http://goelsan.wordpress.com/2010/08/20/design-isa-reflective-practice-a-summary-of-schons-views/.

GONÇALVES, T. AND QUARESMA, P. 2003. A preliminary approach to the multilabel classification problem of Portuguese juridical documents. In Proceedings of the 11th Portuguese Conference on Artificial Intelligence. F. Moura-Pires and S. Abreu Eds., Lecture Notes in Artificial Intelligence, vol. 2902, 435-444.

Greevy, E. AND Smeaton, A. F. 2004. Classifying racist texts using a support vector machine. In Proceedings of the 27th Annual International Conference on Research and Development in Information Retrieval. 468-469.

Hall, M., Frank, E., Holmes, G., Pfahringer, B., Reutemann, P., And Witten, H. 2009. The WeKA data mining software: An update. SIGKDD Explore Newsl.

HAllnäs, L. AND Redström, J. 2001. Slow technology; Designing for reflection. Personal Ubiquit. Comput. 5, 3, 201-212, Springer.

Havasi, C., Speer, R., Pustejovsky, J., And Lieberman, L. 2009. Digital intuition: Applying common sense using dimensionality reduction. IEEE Intelligent Syst. Mag.

Heck, N. C., FlentJe, A., AND Cochran, B. N. 2011. Offsetting risks: High school gay-straight alliances and lesbian, gay, bisexual, and transgender (LGBT) youth. School Psychol. Quart 26, 2, 161-174.

HinduJA, S. AND PATChin, J. 2007. Offline consequences of online victimization: School violence and delinquency. J. School Violence 6, 3, 89-112.

HinduJA, S. AND PATChin, J. W. 2008. Cyberbullying: An exploratory analysis of factors related to offending and victimization. Deviant Behav. 29, 129-156.

IGNIZIO, J. 1991. Introduction to Expert Systems. McGraw-Hill.

JoACHIMS, T. 1998. Text categorization with support vector machines: Learning with many relevant features. In Proceedings of the 10th European Conference on Machine Learning. 137-142.

Kontostathis, A., Edwards, L, And Leatherman, A. 2010. Text mining and cybercrime. In Text Mining: Applications and Theory, M. W. Berry and J. Kogan Eds., John Wiley \& Sons, New York, NY.

LiU, H. AND Singh, P. 2004. ConceptNet - A practical commonsense reasoning tool-kit. BT Technol. J. 22, 4, 211-226.

MAdLOCK, P. AND Westerman, D. 2011. Hurtful cyber-teasing and violence: Who's laughing out loud? J. Interpersonal Violence 26, 17, 3542-3560.

MCNELY, B. 2009. Backchannel persistence and collaborative meaning-making. In Proceedings of the 27th ACM International Conference on Design of Communication. ACM, New York, 297-304.

Menesini, E. AND Nocentini, A. 2009. Cyberbullying definition and measurement: Some critical considerations. J. Psychol. 217, 230-232.

MEY, J. 2001. Pragmatics: An Introduction. Blackwell, 76-77.

Mishna, F., SAini, M., And Solomon, S. 2009. Ongoing and online: Children and youth's perceptions of cyber bullying. Children Youth Services Rev. 31, 12, 1222-1228.

Mishna, F., Cook, C., Gadalla, T., Daciuk, J., And Solomon, S. 2010. Cyber bullying behaviors among middle and high school students. Amer. J. Orthopsychiatry.

Molich, R. And Nielsen, J. 1990. Improving a human-computer dialogue. Comm. ACM 33, 3, 338-348.

National Crime Prevention Council. 2007. Teens and cyberbullying. Executive summary. http://www.ncpc.org/resources/files/pdf/bullying/Teens\%20and\%20Cyberbullying\%20Research $\% 20$ Study.pdf. 
Neviarouskaya, A., Prendinger, H., AND Ishizuka, M. 2009. Compositionality principle in recognition of fine-grained emotions from text. In Proceedings of the International Conference on Weblog and Social Media. AAAI Press, 278-281.

NIELSEN, J. 2000. Why you only need to test with 5 users. http://www.useit.com/alertbox/20000319.html.

NiELSEN, J. 2011. Ten usuabilty heuristics. http://www.useit.com/papers/heuristic/heuristic_list.html.

OL Weus, D. 1993. Bullying at School: What We Know and What We Can Do. Blackwell, Oxford, UK.

Ortony, A., Clore, G. L., And Foss, M. A. 1987. The referential structure of the affective lexicon. Cognitive Sci. 11, 3, 341-364.

Ott, M., Choi, Y., CARDie, C., AND HANCOCK, J. T. 2011. Finding deceptive opinion spam by any stretch of the imagination. In Proceedings of the 49th Annual Meeting of the Association for Computational Linguistics: Human Language Technologies. 309-319.

PANG, B. AND LEE, L. 2008. Opinion mining and sentiment analysis. Found. Trends Info. Retriev. 2, 1-2, $1-135$.

Patchin, J. W. And Hinduja, S. 2012. Cyberbullying Prevention and Response: Expert Perspectives. Routledge, New York.

Quinlan, R. 1999. C4.5: Programs for Machine Learning. Morgan Kaufmann Publishers, San Francisco, CA.

Rogati, M. AND YANG, Y. 2002. High-performing feature selection for text classification. In Proceedings of the 11th International Conference on Information and Knowledge Management.

SASAKI, M. AND KITA, K. 1998. Rule-based text categorization using hierarchical categories. In Proceedings of the IEEE International Conference on Systems, Man, and Cybernetics. Vol. 3, 2827-2830.

SCHÖN D. 1983. The Reflective Practitioner. Basic Books, New York.

SEngers, P. 2005. Reflective design. In Proceedings of the 4th Decennial Conference on Critical Computing. ACM Press, 49-58.

Singh, P., Lin, T., Mueller, T., Lim, G., Perkins, T., And Zhu, W. L. 2002. Open mind common sense: Knowledge acquisition from the general public. In Lecture Notes in Computer Science, vol. 2519, Springer, Berlin, 1223-1237.

Sourander, A., Klomek, A. B., Ikonen, M., Lindroos, J., Luntamo, T., Koskelainen, M., RistKaRI, T., AND Helenius, H. 2010. Psychosocial risk factors associated with cyberbullying among adolescents: A population-based study. Arch Gen Psychiatry.

Speer, R., Havasi, C., AND Lieberman, H. 2008. AnalogySpace: Reducing the imensionality of common sense knowledge. In Proceedings of the 23rd National Conference on Artificial Intelligence. Vol. 1, A. Cohn Ed., AAAI Press, 548-553.

Stop Cyberbullying. 2011. How to handle a cyberbully. http://www.stopcyberbullying.org/parents/howdoyouhandleacyberbully.html.

TONG, S. AND KOLLER, D. 2000. Support vector machine active learning with applications to text classification. In Proceedings of the 17th International Conference on Machine Learning. 999-1006.

Vala, M., Sequeira, P., Paiva, A., And Aylett, R. 2007. FearNot! demo: A virtual environment with synthetic characters to help bullying. In Proceedings of the 6th International Joint Conference on Autonomous Agents and Multiagent Systems.

Vandebosch H. ANd Cleemput, K. V. 2009. Cyberbullying among youngsters: Profiles of bullies and victims. New Media \& Society 11, 1349-1371.

WALL. 2011. How to use the Wall feature and Wall privacy. http://www.facebook.com/help/?page=174851209237562.

WALther, J. B., LOH, T., AND GRANKA, L. 2005. Let me count the ways: The interchange of verbal and nonverbal cues in computer-mediated and face-to-face affinity. J. Lang. Social Psychol. 24, 36-65.

YBARRA, M. 2010. Trends in technology-based sexual and non-sexual aggression over time and linkages to non-technology aggression. National Summit on Interpersonal Violence and Abuse Across the Lifespan: Forging a Shared Agenda.

Received May 2011; revised December 2011; accepted March 2012 